\title{
Modelling and QoS-Achieving Solution in full-duplex Cellular Systems
}

\author{
Ali Y. Al-Zahrani \\ Department of Electrical and Computer Engineering \\ University of Jeddah \\ Jeddah 21589, Saudi Arabia
}

\section{ABSTRACT}

The global bandwidth scarcity and the ever-growing demand for fast wireless services have motivated the quest for new techniques that enhance the spectral efficiency (SE) of wireless systems. Most conventional SE increasing methods (e.g., adaptive modulation and coding) have already been exhausted. Single-channel full-duplex (SCFD) communication is a new attractive approach in which each node may simultaneously receive and transmit over the same frequency channel, and thus, it has the potential to double the current SE figures. In this paper, we derive a model for the signal-to-interference-plus-noise ratio (SINR) in a SCFD-based cellular system with imperfect self-interference cancellation. Furthermore, given a set of uplink and downlink quality of service requirements, we answer the following two fundamental questions. First, is this set achievable in the SCFD-based cellular system? Second, if the given set is achievable, what is the optimal achieving policy? To that end, we provide a unified model for the SCFD-based cellular system, and give insights in the matrix of interference channel gains. Simulation results suggest that depending on the locations of the users, a combination of full-duplex and half-duplex modes over the whole network is more favourable policy.

$\boldsymbol{K} \boldsymbol{E} \boldsymbol{Y} \boldsymbol{W O R} \boldsymbol{D} \boldsymbol{S}$ - full-duplex, cellular network, self-interference, inter-cell interference, resource allocations and interference cancellation 
International Journal of Computer Networks \& Communications (IJCNC) Vol.10, No.5, September 2018

\section{INTRODUCTION}

The ever-increasing demand for wireless services stresses on finding new methods that increase the spectral efficiency. A recent method to enhance the spectral efficiency is called single-channel full-duplex (SCFD) transmission. In SCFD, any wireless device is permitted to simultaneously receive and transmit information over the same frequency channel. Traditionally, devices are able to transmit and receive either at different instants or over different frequency bands [1].

Although SCFD transmission has the potential to double the spectral efficiency [2], its successful implementation faces a major technical difficulty, that is selfinterference problem. self-interference is the interference caused by the terminal on itself due to in-band and simultaneous transmission and reception (i.e., transmitting a high power signal and receiving a weak power desired signal) $[2,3]$.

self-interference can be classified according to its path into two types: direct-path self-interference in which the transmit signal propagates directly from the transmitting antenna to the receiving antenna, and reflected-path self-interference in which the transmit signal is reflected by the surrounding environment to the receiving antenna $[4,5]$. Canceling the former is relatively simple using propagation-domain techniques such as antenna polarization and antenna directionality [3,6-9] whereas the latter needs additionally more sophisticated adaptive methods in both analog and digital domains [10-16]. The degree of complexity depends on the bandwidth of the transmit signal. In general, the narrower the signal's bandwidth is the simpler a design would be.

Since the transmitted signal is known to the terminal, one might suggest canceling the self-interference using any digital cancellation technique [19]. Although the digital baseband interference cancellation helps alleviate the self-interference, it is actually insufficient. The analog circuits in the front end of the transceiver and the limited dynamic range of the analog-to-digital converter (ADC) distort the transmitted signals. Thus, the digital interference cancellation becomes imperfect due to the difference between the original signal and its distorted copy. Despite the progress in the antenna designs and analog/digital cancellation techniques [3,6-16], the selfinterference is still above the noise floor, and hence can affect the detection of the desired received signals. Therefore, the goal of completely doubling the spectral efficiency is hard to achieve without a tight interference control.

Because SCFD eliminates the in-band half-duplex constraint, which is a fundamental constraint in the conventional wireless networks [1], modeling and redesigning many aspects of wireless networks must be considered in case of SCFD. In this paper, we model the problem of inter-cell interference in multi-cell SCFD wireless systems 
with imperfect self-interference cancellation. Then, we redesign the inter-cell interference control techniques under certain quality of service (QoS) requirements.

In cellular networks, there are two types of wireless full-duplex communications: symmetric and asymmetric [2,21]. In symmetric full-duplex communication, the full-duplex transmission occurs between two nodes (usually the base station (BS) and one user equipment (UE)). This kind of SCFD is sometimes called bidirectional. On the other hand, the asymmetric half-duplex includes three nodes (usually BS, downloader UE and uploader UE). In this research, we propose a unified model that captures both kinds of SCFD $[2,22]$.

In half-duplex transmission-based networks, spectrum sharing as well as interference management and power control have been studied very well. References $[20,23,24]$, for example, studied these issues in latest applications such as heterogeneous and device to device (D2D) networks. On the other hand, the area of SCFDbased wireless networks has just got enough attention that produced some constructive research results. However, very few papers in this domain consider large SCFDbased cellular systems with imperfect self-interference cancellation. The authors in [25] studied the end-to-end capacity of SCFD relaying with two modes: amplifyand-forward and decode-and-forward. They evaluated the self-interference threshold below which the full-duplex transmission outperforms the half-duplex transmission. In their work, they considered the isolated system of 3 nodes only, that is one link of two hops (source-relay-destination). In [26], a single cell full-duplex system aided with the multi-input-multi-output (MIMO) technology was considered. The authors exploited the large number of antennas at the BS for self-interference cancellation. Optimal dynamic power allocation schemes for different scenarios were developed in [4] with an objective of maximizing the sum rate of a SCFD bidirectional link. However, the inter-cell interference was not considered. In [21], a simple decodeand-forward full-duplex MIMO relaying, the transmitter/receiver dynamic range limitations were modeled. More importantly, a tight upper and lower bounds on the end-to-end achievable rate were derived. In addition, the authors proposed a transmission policy that maximizes the lower bound.They also derived a theoretical approximation of the achievable rate which helps to gain insights into the system design tradeoffs. The authors in [22] analyzed the effects of adopting SCFD enabled BSs in an OFDM multicell network with legacy UEs. However, in their work, the perfect self-interference cancellation was assumed. In [17] the unique advantages of FD radios are identified and the multi-input multi- output (MIMO) communication is leveraged to translate the FD spectral efficiency gain at the PHY level to throughput and power efficiency gain at the network layer. The authors in [18] studied two scenarios of full-duplex (FD) multiple-input?multiple-output cognitive radio networks: 


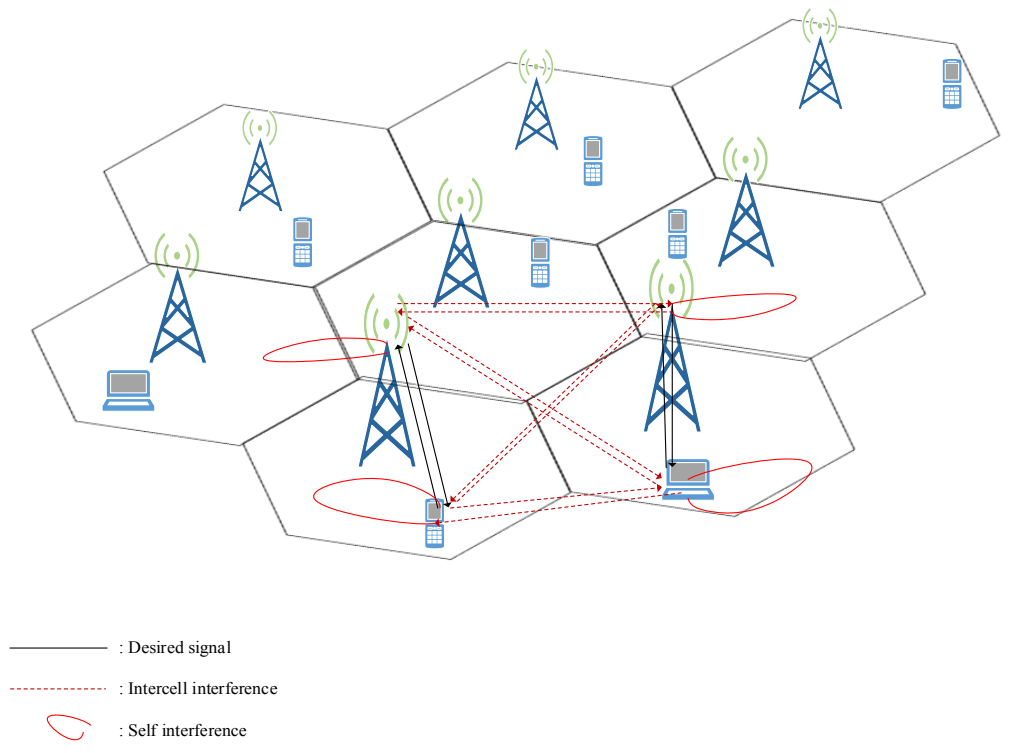

Figure 1: A full-duplex cellular network

FD cognitive ad hoc networks and FD cognitive cellular networks. Two optimization problems were addressed: one is to minimize the sum of mean-squared errors (MSE) of all estimated symbols whereas the other is to minimize the maximum MSE of estimated symbols, both problems are subject to power constraints at the secondary users and interference constraints projected to each primary users

The rest of this paper is organized as follows. In the next section, the setup of the full-duplex cellular system is developed, and the mathematical model of a crucial performance metric (SINR) is derived. In section 3, the feasibility of a given set of target SINRs is addressed, and an optimal achieving policy is provided. Finally, the conclusion of this research and possible future directions are discussed in section 5 .

\section{FUlL-DUPleX System Model}

We consider $N$ full-duplex links each of which is located in a separate cell as shown in Figure 1. The transmitter typically sends a signal $x(t)$ to its intended receiver as shown in Fig.(2). However, this signal is distorted by the RF analog circuits in the transmitter front end. Moreover, after passing through the channel and corrupted by the noise and different interfering signals, the pre-ADC received signal $u(t)$ impinging the receiver antenna is distorted again by $\mathrm{RF}$ analog circuits and the ADC 


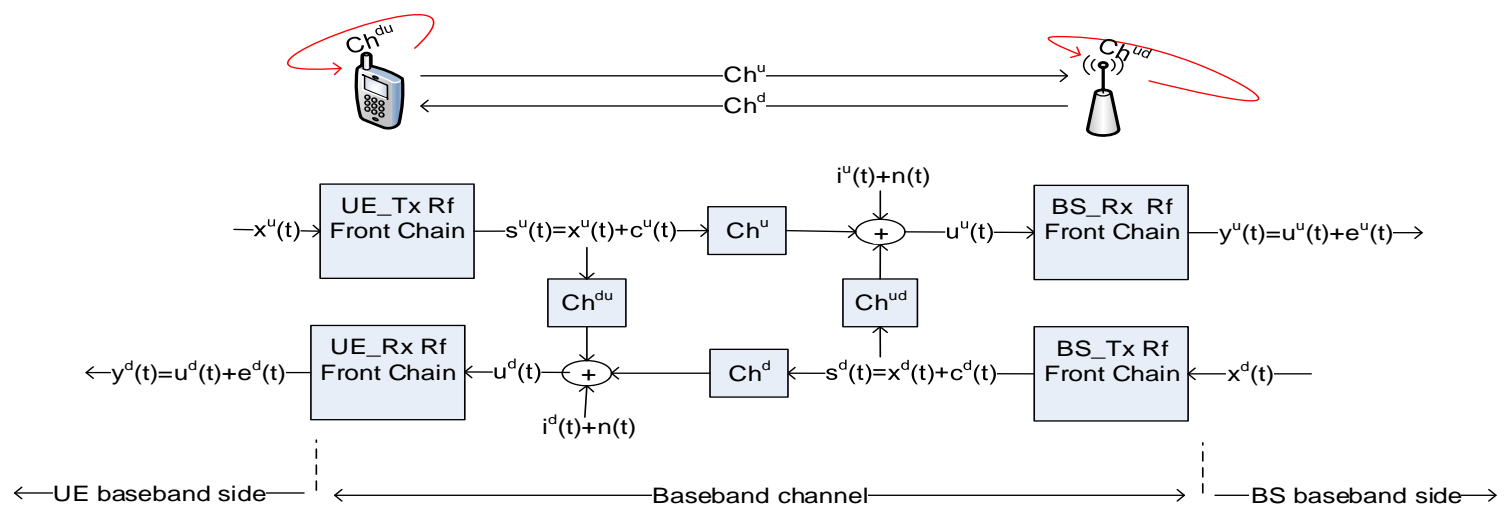

Figure 2: A full-duplex baseband-equivalent system for both uplink and downlink signals

in the receiver front end.Figure 2 shows the discrete-time baseband-equivalent model for full-duplex (FD) link. This model, which is closely related to the model proposed in [21], captures the effects of analog circuits and the limited dynamic range in both sides of the link. The effect of the transmitter distortion is captured by injecting an independent zero-mean Gaussian noise $c(t)$ whose variance equals the power of the intended signal $x(t)$ times a constant parameter $\kappa$. This noise closely approximates the combined effects of additive power-amp harmonics, non-linearities in the digital-to-analog converter (DAC) and power-amp, and oscillator phase noise [21]. Therefore, the baseband equivalent of the radiated signal is

$$
s(t)=x(t)+c(t) ; \text { suchthat }\left\{\begin{array}{l}
c(t) \sim \mathcal{N}\left(0, \kappa|x(t)|^{2}\right) \\
c(t) \perp x(t) \\
c(t) \text { is white. }
\end{array}\right.
$$

On the other hand, the effect of the receiving RF front end and the ADC is captured by injecting an independent zero-mean Gaussian noise $e(t)$ whose variance equals the power of the pre-ADC received signal $u(t)$ times a constant parameter $\beta$. Therefore, the baseband signal after ADC would be

$$
y(t)=u(t)+e(t) ; s . t .\left\{\begin{array}{l}
e(t) \sim \mathcal{N}\left(0, \beta|u(t)|^{2}\right) \\
e(t) \perp u(t) \\
e(t) \text { is white. }
\end{array}\right.
$$

Note that $\kappa \ll 1$ and $\beta \ll 1$ are system parameters. The pre-ADC received signal $u(t)$ includes the desired signal, self-interference and inter-cell interference plus noise. 
Thus, the pre-ADC received uplink and downlink signals at the $n^{\text {th }}$ BS antenna and its associated UE antenna would be respectively as follows

$$
\begin{aligned}
& u_{n}^{u}(t)=h_{n n}^{u}(t)\left(x_{n}^{u}(t)+c_{n}^{u}(t)\right)+h_{n n}^{u d}\left(x_{n}^{d}(t)+c_{n}^{d}(t)\right)+i_{n}^{u}(t)+n_{n}^{u}(t) \\
& u_{n}^{d}(t)=h_{n n}^{d}(t)\left(x_{n}^{d}(t)+c_{n}^{d}(t)\right)+h_{n n}^{d u}\left(x_{n}^{u}(t)+c_{n}^{u}(t)\right)+i_{n}^{d}(t)+n_{n}^{d}(t) ;
\end{aligned}
$$

where the superscript $d$ and $u$ indicate downlink and uplink, respectively. $h_{n n}^{d}(t)$ and $h_{n n}^{u}$ are the desired downlink and uplink channel responses. $h_{n n}^{d u}$ is the channel response of the uplink to downlink self-interference (i.e., self-interference at UE) whereas $h_{n n}^{u d}$ is the channel response of the downlink to uplink self-interference(i.e., self-interference at BS). The additive white Gaussian noise processes at both receiving ends are represented by $n_{n}^{u}(t)$ and $n_{n}^{d}(t)$. Finally, $i_{n}^{u}(t)$ and $i_{n}^{d}(t)$ are the inter-cell interference signals impinging the antennas of the $n^{\text {th }}$ BS and its associated UE, respectively. They can be expressed as

$$
\begin{aligned}
i_{n}^{d}(t) & =\sum_{\substack{k \in \mathcal{N} \\
k \neq n}}\left(h_{n k}^{d d}(t) s_{k}^{d}(t)+h_{n k}^{d u} s_{k}^{u}(t)\right) \\
i_{n}^{u}(t) & =\sum_{\substack{k \in \mathcal{N} \\
k \neq n}}\left(h_{n k}^{u d}(t) s_{k}^{d}(t)+h_{n k}^{u u} s_{k}^{u}(t)\right) .
\end{aligned}
$$

Using equations (2) and (3), the received baseband signals at both ends would then be (after some arrangement)

$$
\begin{aligned}
& y_{n}^{d}(t)=\underbrace{h_{n n}^{d}(t) x_{n}^{d}(t)}_{\text {Desired signal }}+\underbrace{h_{n n}^{d u} x_{n}^{u}(t)}_{\text {self-interference }}+\underbrace{h_{n n}^{d}(t) c_{n}^{d}(t)+h_{n n}^{d u} c_{n}^{u}(t)+e_{n}^{d}+n_{n}^{d}(t)}_{\text {Aggregate noise due to Rf front ends distortions }}+i_{n}^{d}(t) ; \\
& y_{n}^{u}(t)=\underbrace{h_{n n}^{u}(t) x_{n}^{u}(t)}_{\text {Desired signal }}+\underbrace{h_{n n}^{u d} x_{n}^{d}(t)}_{\text {self-interference }}+\underbrace{h_{n n}^{u}(t) c_{n}^{u}(t)+h_{n n}^{u d} c_{n}^{d}(t)+e_{n}^{u}+n_{n}^{u}(t)}_{\text {Aggregate noise due to Rf front ends distortions }}+i_{n}^{u}(t) .
\end{aligned}
$$

At every cell, we assume that the desired channels and the self-interference channels are estimated correctly. Therefore, the first term in the above two equations is the desired signal. The self-interference in the second term is known and thus can be canceled. The third term captures the aggregate distortions introduced by the front end chains. The last term is the inter-cell interference. 
After self-interference cancellation ${ }^{1}$, the SINR at both ends can thus be written as follows(suppressing the argument $(\mathrm{t})$ )

$$
\begin{aligned}
\gamma_{n}^{d} & = \begin{cases}\frac{p_{n}^{d} g_{n n}^{d}}{\kappa p_{n}^{d} g_{n n}^{d}+\kappa p_{n}^{u} g_{n n}^{d u}+\beta\left|u_{n}^{d}\right|^{2}+\sigma_{n_{n}^{d}}^{2}+I_{n}^{d}} & \text { for symmetric communication } \\
\frac{p_{n}^{d} g_{n n}^{d}}{\kappa p_{n}^{d} g_{n n}^{d}+(1+\kappa) p_{n}^{u} g_{n n}^{d u}+\beta\left|u_{n}^{d}\right|^{2}+\sigma_{n_{n}^{d}}^{2}+I_{n}^{d}} & \text { for asymmetric communication }\end{cases} \\
\gamma_{n}^{u} & =\frac{p_{n}^{u} g_{n n}^{u}}{\kappa p_{n}^{u} g_{n n}^{u}+\kappa p_{n}^{d} g_{n n}^{u d}+\beta\left|u_{n}^{u}\right|^{2}+\sigma_{n_{n}^{u}}^{2}+I_{n}^{u}}
\end{aligned}
$$

where $p_{n}^{d}=\left|x_{n}^{d}\right|^{2}$ and $p_{n}^{u}=\left|x_{n}^{u}\right|^{2}$ are the downlink and uplink transmit power, respectively. $g=|h|^{2}$ is generally the power gain of a given channel $h$. While $\sigma_{n_{n}}^{2}$ is the additive noise power, $I_{n}^{d}$ and $I_{n}^{u}$ are the total inter-cell interference power affecting the downlink signal and the uplink signal, respectively. According to (3), $\beta\left|u_{n}^{d}\right|^{2}$ and $\beta\left|u_{n}^{u}\right|^{2}$ can be evaluated as follows

$$
\begin{aligned}
& \beta\left|u_{n}^{d}\right|^{2}=\beta\left[(1+\kappa) p_{n}^{d} g_{n n}^{d}+(1+\kappa) p_{n}^{u} g_{n n}^{d u}+\sigma_{n_{n}^{d}}^{2}+I_{n}^{d}\right] \\
& \beta\left|u_{n}^{u}\right|^{2}=\beta\left[(1+\kappa) p_{n}^{u} g_{n n}^{u}+(1+\kappa) p_{n}^{d} g_{n n}^{u d}+\sigma_{n_{n}^{u}}^{2}+I_{n}^{u}\right] .
\end{aligned}
$$

In the last two equations, since $\kappa \ll 1$ and $\beta \ll 1$, any term which includes $\kappa \beta$ is considered significantly small, and hence it could be ignored. Therefore, the SINR of both links is updated in the following equations

$$
\begin{aligned}
\gamma_{n}^{d} & =\frac{p_{n}^{d} g_{n n}^{d}}{(\kappa+\beta) p_{n}^{d} g_{n n}^{d}+\left(P_{I}+\kappa+\beta\right) p_{n}^{u} g_{n n}^{d u}+(1+\beta) \sigma_{n_{n}^{d}}^{2}+(1+\beta) I_{n}^{d}} \\
\gamma_{n}^{u} & =\frac{p_{n}^{u} g_{n n}^{u}}{(\kappa+\beta) p_{n}^{u} g_{n n}^{u}+(\kappa+\beta) p_{n}^{d} g_{n n}^{u d}+(1+\beta) \sigma_{n_{n}^{u}}^{2}+(1+\beta) I_{n}^{u}}
\end{aligned}
$$

where $P_{I}$ is an indicator parameter which equals 0 in case of symmetric communications and 1 for asymmetric communications. Since $g_{n n}^{d} \ll g_{n n}^{u d}$ due to the incurred path loss, the distortion $(\kappa+\beta) p_{n}^{d} g_{n n}^{d}$ is usually ignored in all previously conducted research on conventional half-duplex systems, with an implicit assumption that this distortion arrives very faded and at the noise floor. Therefore, this part of distortion may be regarded as part of the additive noise: $(\kappa+\beta) p_{n}^{d} g_{n n}^{d}+(1+\beta) \sigma_{n_{n}^{d}}^{2}=\sigma_{o}^{2}$. A similar argument can be made on the uplink. Thus, the SINR at both ends can be

\footnotetext{
${ }^{1}$ In case of asymmetric communication (i.e., one UE transmits an uplink stream to the BS, while another UE receives a downlink stream from the BS), then only BS can perform self-interference cancellation, and the downlink user will regard the signal from the uplink user as an interference.
} 
International Journal of Computer Networks \& Communications (IJCNC) Vol.10, No.5, September 2018

rewritten as

$$
\begin{aligned}
\gamma_{n}^{d} & =\frac{p_{n}^{d} g_{n n}^{d}}{(1+\beta) I_{n}^{d}+\left(P_{I}+\kappa+\beta\right) p_{n}^{u} g_{n n}^{d u}+\sigma_{o}^{2}} \\
\gamma_{n}^{u} & =\frac{p_{n}^{u} g_{n n}^{u}}{(1+\beta) I_{n}^{u}+(\kappa+\beta) p_{n}^{d} g_{n n}^{u d}+\sigma_{o}^{2}}
\end{aligned}
$$

This model includes the case of perfect self-interference cancellation. Authors in [15] claimed that they cancelled the self-interference to the noise level. In this case, $\kappa, \beta=0$.

\section{A Required QoS: Feasibility and Solutions}

Any required quality of service (QoS) which basically consists of the minimum required data rate and the maximum probability of error, can be mapped into a certain SINR target. This section derives a criterion on whether a given set of target SINRs is achievable or not. To that end, we will first consider the case of one cell network (i.e., $N=1$ ), afterward, a multi-cell case will then be derived by extension.

\subsection{Single-Cell system}

In the case of a single-cell full-duplex wireless system, $I^{d}$ and $I^{u}$ are considered of zero values. Let $\Gamma^{d}$ and $\Gamma^{u}$ be the target SINRs required for the downlink stream and uplink stream, respectively. We are interested to answer two questions: 1) Is this pair of required SINRs feasible (i.e., achievable)? 2) If it is feasible, what is the best policy for achieving it? To answer these questions, let's start from a basic objective inequality in which the actual received SINRs at both ends are required to be greater than or equal to the target SINRs

$$
\begin{aligned}
& \gamma^{d} \geq \Gamma^{d}, \\
& \gamma^{u} \geq \Gamma^{u}
\end{aligned}
$$

Substituting (9) into (10) produces

$$
\begin{aligned}
& p^{d} \geq \frac{\Gamma^{d}}{g^{d}}\left(\left(P_{I}+\kappa+\beta\right) p^{u} g^{d u}+\sigma_{o}^{2}\right), \\
& p^{u} \geq \frac{\Gamma^{u}}{g^{u}}\left((\kappa+\beta) p^{d} g^{u d}+\sigma_{o}^{2}\right) .
\end{aligned}
$$


The above system of inequalities in (11) can be expressed in a matrix form as follows

$$
\mathbf{p} \geq \mathbf{D G p}+\mathbf{D} \sigma,
$$

where $\mathbf{p}=\left[\begin{array}{ll}p^{d} & p^{u}\end{array}\right]^{T} \geq \mathbf{0}$. The $2 \times 2$ matrix $\mathbf{D}$ is a diagonal matrix which is equal to $\operatorname{diag}\left(\frac{\Gamma^{d}}{g^{d}}, \frac{\Gamma^{u}}{g^{u}}\right)$, whereas $\mathbf{G}$ is the self-interference channel gain matrix, that is:

$$
\mathbf{G}=\left[\begin{array}{cc}
0 & (P+\kappa+\beta) g^{d u} \\
(\kappa+\beta) g^{u d} & 0
\end{array}\right] .
$$

Finally, the noise vector equals $\sigma=\left[\begin{array}{ll}\sigma_{o}^{2} & \sigma_{o}^{2}\end{array}\right]^{T}$.

The central solution of (12) is

$$
\mathbf{p} \geq[\mathbf{I}-\mathbf{D G}]^{-1} \mathbf{D} \sigma \text {, subject to } \mathbf{p} \geq \mathbf{0},
$$

where $\mathbf{I}$ is the identity matrix. Notice that $\mathbf{D G}$ is a nonnegative matrix, and hence the spectral radius ${ }^{2} \rho(\mathbf{D G})$ is an eigenvalue of DG [27]. According to the PerronFrobenius theory [27], the solution in (14) is a nonnegative transmit power vector if and only if the spectral radius $\rho(\mathbf{D G})$ is less than unity, mathematically,

$$
[\mathbf{I}-\mathbf{D G}]^{-1} \mathbf{D} \sigma \geq 0 \Leftrightarrow \rho(\mathbf{D G})<1 .
$$

Satisfying the nonnegative power constraint in (14) actually depends on the values of $\Gamma^{d}$ and $\Gamma^{u}$. Therefore, the pair of target SINRs is feasible iff $\rho(\mathbf{D G})<1$.

The solution $\mathbf{p}^{*}=[\mathbf{I}-\mathbf{D G}]^{-1} \mathbf{D} \sigma$ is Pareto optimal since all other feasible solutions require at least same power element-wise (i.e., $\mathbf{p} \geq \mathbf{p}^{*} \forall \mathbf{p} \geq 0$ satisfying (14)). The policy in one cell system is as follows. Over the control channel, the UE sends the BS the uplink target SINR $\Gamma^{u}$ and the estimated channel gain of the self-interference $g^{d u}$. Since downlink and uplink transmissions occur over the same frequency band, the downlink and uplink channels are reciprocal, and hence, $g^{d}, g^{u}$ can be easily estimated at the BS. Using $\mathbf{p}^{*}=[\mathbf{I}-\mathbf{D G}]^{-1} \mathbf{D} \sigma$, the BS computes the best transmit power for both uplink and downlink transmissions. Then, it dictates its associated UE to act accordingly.

\subsection{Multicell System}

In a system of $N$ cells, the required QoS for both uplink and downlink streams in the $n^{\text {th }}$ cell can be mapped into a pair of target SINRs $\Gamma_{n}^{d}$ and $\Gamma_{n}^{u}$. To achieve the 
International Journal of Computer Networks \& Communications (IJCNC) Vol.10, No.5, September 2018

$$
\begin{aligned}
& \frac{p_{n}^{d} g_{n n}^{d}}{(1+\beta) \sum_{\substack{k \in \mathcal{N} \\
k \neq n}} g_{n k}^{d d} p_{k}^{d}+(1+\beta) \sum_{\substack{k \in \mathcal{N} \\
k \neq n}} g_{n k}^{d u} p_{k}^{u}+\left(P_{I}+\kappa+\beta\right) p_{n}^{u} g_{n n}^{d u}+\sigma_{o}^{2}} \geq \Gamma_{n}^{d}, \quad \forall n \in \mathcal{N} \\
& \frac{p_{n}^{u} g_{n n}^{u}}{(1+\beta) \sum_{\substack{k \in \mathcal{N} \\
k \neq n}} g_{n k}^{u d} p_{k}^{d}+(1+\beta) \sum_{\substack{k \in \mathcal{N} \\
k \neq n}} g_{n k}^{u u} p_{k}^{u}+(\kappa+\beta) p_{n}^{d} g_{n n}^{u d}+\sigma_{o}^{2}} \geq \Gamma_{n}^{u}, \quad \forall n \in \mathcal{N} .
\end{aligned}
$$

target QoS for both streams, the actual SINRs of both links have to equal at least the target required SINRs $\Gamma_{n}^{d}$ and $\Gamma_{n}^{u}$; that is,

Note that the first two terms in the denominators account for inter-cell interference. These two inequalities can be compacted in a matrix form that models the whole system

$$
\mathrm{p} \geq \mathrm{DGp}+\nu,
$$

where $\mathbf{p}=\left[\begin{array}{ll}\mathbf{p}^{d} & \mathbf{p}^{u}\end{array}\right]^{T}=\left[p_{1}^{d}, \ldots, p_{N}^{d}, p_{1}^{u}, \ldots, p_{N}^{u}\right]^{T}$ is the vector of all downlink and uplink transmit powers. The $2 N \times 2 N$ matrix $\mathbf{D}$ is a diagonal matrix that equals $(1+\beta) \operatorname{diag}\left(\frac{\Gamma_{1}^{d}}{g_{11}^{d}}, \ldots, \frac{\Gamma_{N}^{d}}{g_{N N}^{d}}, \frac{\Gamma_{1}^{u}}{g_{11}^{u}}, \ldots, \frac{\Gamma_{N}^{u}}{g_{N N}^{u}}\right)$ while $\boldsymbol{\nu}=\left[\begin{array}{ll}\boldsymbol{\nu}^{d} & \boldsymbol{\nu}^{u}\end{array}\right]^{T}$ is a normalized noise vector, where $\boldsymbol{\nu}^{d}=\left[\frac{\Gamma_{1}^{d} \sigma_{o}^{2}}{g_{11}^{d}}, \ldots ., \frac{\Gamma_{N}^{d} \sigma_{o}^{2}}{g_{N N}^{d}}\right]$ and $\boldsymbol{\nu}^{u}=\left[\frac{\Gamma_{1}^{u} \sigma_{o}^{2}}{g_{11}^{u}}, \ldots ., \frac{\Gamma_{N}^{u} \sigma_{o}^{2}}{g_{N N}^{u}}\right]$. The $2 N \times 2 N$ matrix $\mathbf{G}$ is the gains matrix of the interference channels whose diagonal elements equal to zero, and can be simplified as

$$
\mathbf{G}=\left[\begin{array}{ll}
\mathbf{G}^{d d} & \mathbf{G}^{d u} \\
\mathbf{G}^{u d} & \mathbf{G}^{u u}
\end{array}\right],
$$

where the zero-diagonal submatricies $\mathbf{G}^{d d}$ and $\mathbf{G}^{u u}$ are the downlink to downlink interference channel gain and uplink to uplink interference channel gain, respectively. Moreover, $\mathbf{G}^{d u}$ represents the uplink to downlink interference channel gains whose diagonal is the normalized self-interference: $\frac{\left(P_{I}+\kappa+\beta\right) g_{n n}^{d u}}{1+\beta}$, for $n=1 \ldots N$. Finally, $\mathbf{G}^{u d}$ represents the downlink to uplink interference channel gains whose diagonal is the normalized self-interference: $\frac{(\kappa+\beta) g_{n n}^{u d}}{1+\beta}$, for $n=1 \ldots N$. In case of a sophisticated selfinterference cancellation that the residual interference is brought down to the noise level, then the diagonal elements of $\mathbf{G}^{d u}$ and $\mathbf{G}^{u d}$ are equal to zero. A breakdown of

\footnotetext{
${ }^{2}$ Spectral radius of a matrix $\mathbf{A}$ is the maximum absolute value of $\mathbf{A}$ 's eigenvalue elements (i.e., $\left.\rho(\mathbf{A})=\max \left\{\left|\lambda_{i}\right|\right\}_{i=1}^{i=N}\right)$.
} 
$\mathbf{G}$ is shown in (19).

$D\left\{\left[\begin{array}{cccccccc}\overbrace{0} & g_{12}^{d d} & \ldots & g_{1 N}^{d d} & \overbrace{\frac{\left(P_{I}+\kappa+\beta\right) g_{11}^{d u}}{1+\beta}}^{D} & g_{12}^{d u} & \ldots & g_{1 N}^{d u} \\ g_{21}^{d d} & 0 & \ldots & g_{2 N}^{d d} & g_{21}^{d u} & \frac{\left(P_{I}+\kappa+\beta\right) g_{22}^{d u}}{1+\beta} & \ldots & g_{2 N}^{d u} \\ \vdots & \vdots & \ddots & \vdots & \vdots & \vdots & \ddots & \vdots \\ g_{N 1}^{d d} & g_{N 2}^{d d} & \ldots & 0 & g_{N 1}^{d u} & g_{N 2}^{d u} & \ldots & \frac{\left(P_{I}+\kappa+\beta\right) g_{N N}^{d u}}{1+\beta} \\ \frac{(\kappa+\beta) g_{11}^{u d}}{1+\beta} & g_{12}^{u d} & \ldots & g_{1 N}^{u d} & 0 & g_{12}^{u u} & \ldots & g_{1 N}^{u u} \\ g_{21}^{u d} & \frac{(\kappa+\beta) g_{22}^{u d}}{1+\beta} & \ldots & g_{2 N}^{u d} & g_{21}^{u u} & 0 & \ldots & g_{2 N}^{u u} \\ \vdots & \vdots & \ddots & \vdots & \vdots & \vdots & \ddots & \vdots \\ g_{N 1}^{u d} & g_{N 2}^{u d} & \ldots & \frac{(\kappa+\beta) g_{22}^{u d}}{1+\beta} & g_{N 1}^{u u} & g_{N 2}^{u u} & \ldots & 0\end{array}\right]\right.$.

Since all nodes transmit over the same frequency channel, the channel between any two nodes can be considered reciprocal (e.g., $h_{i j}^{d u}=h_{j i}^{d u}$ ). Therefore, $\mathbf{G}^{d d}=$ $\left[\mathbf{G}^{u u}\right]^{T}$ and $\mathbf{G}^{u d}$ is a symmetric matrix whereas $\mathbf{G}^{d u}$ is symmetric matrix only in case of symmetric communications. Thus,

$$
\mathbf{G}^{T}=\left[\begin{array}{ll}
\mathbf{G}^{u u} & \mathbf{G}^{u d} \\
\mathbf{G}^{d u} & \mathbf{G}^{d d}
\end{array}\right]
$$

Note that $\mathbf{G}$ is irreducible matrix, and $\mathbf{D G}$ is a nonnegative matrix. Below we rewrite (17) as

$$
\mathbf{p} \geq[\mathbf{I}-\mathbf{D G}]^{-1} \boldsymbol{\nu}
$$

where, $\mathbf{I}$ is the identity matrix. Applying Perron-Frobenius theory, the target SINRs $\Gamma$ can be achieved with nonnegative solution $\mathbf{p}^{*}$ if and only if the spectral radius $\rho(\mathbf{D G})$ is less than unity, mathematically,

$$
\mathbf{p}^{*}=[\mathbf{I}-\mathbf{D G}]^{-1} \boldsymbol{\nu} \geq \mathbf{0} \Leftrightarrow \rho(\mathbf{D G})<1
$$

Accordingly, if the set of target SINRs is feasible, then $\mathbf{p}^{*}=[\mathbf{I}-\mathbf{D G}]^{-1} \boldsymbol{\nu}$ represents the centralized policy which requires all system's information to be collected at a central entity for processing. This is obviously impractical in a large multi-cell system. In this case, we should find a distributed solution that ends up with the same 
International Journal of Computer Networks \& Communications (IJCNC) Vol.10, No.5, September 2018

solution as that of the centralized one.

Notice that (17) (with equality) represents the iterative distributed solution using the best response algorithm, i.e.,

$$
\mathbf{p}(\tau+1)=\operatorname{DGp}(\tau)+\nu
$$

In other words, the behaviour of the nodes in cell $n$ is

$$
\begin{array}{r}
p_{n}^{d}(\tau+1)=\Gamma_{n}^{d} \frac{(1+\beta) I_{n}^{d}(\tau)+\left(P_{I}+\kappa+\beta\right) p_{n}^{u}(\tau) g_{n n}^{d u}+\sigma_{o}^{2}}{g_{n n}^{d}} \\
p_{n}^{u}(\tau+1)=\Gamma_{n}^{u} \frac{(1+\beta) I_{n}^{u}(\tau)+(\kappa+\beta) p_{n}^{d}(\tau) g_{n n}^{u d}+\sigma_{o}^{2}}{g_{n n}^{u}}
\end{array}
$$

Equivalently, this can be written ${ }^{3}$ as:

$$
\begin{aligned}
& p_{n}^{d}(\tau+1)=\Gamma_{n}^{d} \frac{p_{n}^{d}(\tau)}{\gamma_{n}^{d}(\tau)} \\
& p_{n}^{u}(\tau+1)=\Gamma_{n}^{u} \frac{p_{n}^{u}(\tau)}{\gamma_{n}^{u}(\tau)}
\end{aligned}
$$

Thus, plugging the current transmit power levels and the current measured SINRs in the above equations will generate the required transmit power in the next iteration $(\tau+1)$.

Let the right hand side $(\mathrm{RHS})$ of $(17)$ be $F(\mathbf{p})=\mathbf{D G p}+\boldsymbol{\nu}$. Then, the solution of $(23)$ is actually the fixed point of $F(\mathbf{p})$. Moreover, $F(\mathbf{p})$ possesses the following properties:

- positivity i.e., $F(\mathbf{p})>0 \forall \mathbf{p} \geq 0$,

- monotonicity i.e., if $\mathbf{p}_{1}>\mathbf{p}_{2} \Rightarrow F\left(\mathbf{p}_{1}\right)>F\left(\mathbf{p}_{2}\right)$,

- scalability i.e., for any $a>1, a F(\mathbf{p})>F(a \mathbf{p})$.

According to Yates [28], $F(\mathbf{p})$ is called a standard interference function. In addition, if the problem is feasible, then $F(\mathbf{p})$ has a unique fixed point. Furthermore, as the iteration count $\tau \rightarrow \infty$, the power vector $\mathbf{p}$ in (23) converges to the unique fixed point $\mathbf{p}^{*}$. Therefore, the distributed algorithm in (23) will surely converge to the Pareto optimal solution.

\footnotetext{
${ }^{3}$ This is done by multiplying the denominator and numerator by the corresponding transmit power $p_{n}(\tau)$.
} 
International Journal of Computer Networks \& Communications (IJCNC) Vol.10, No.5, September 2018

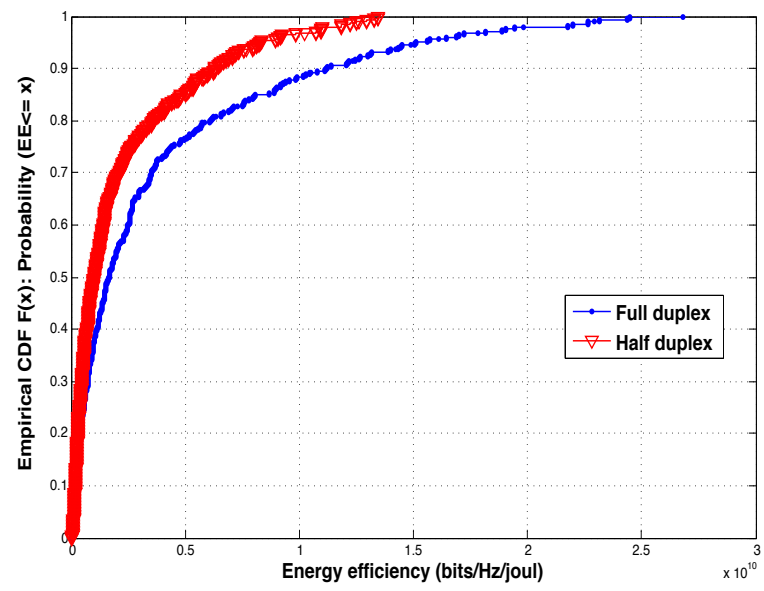

(a)

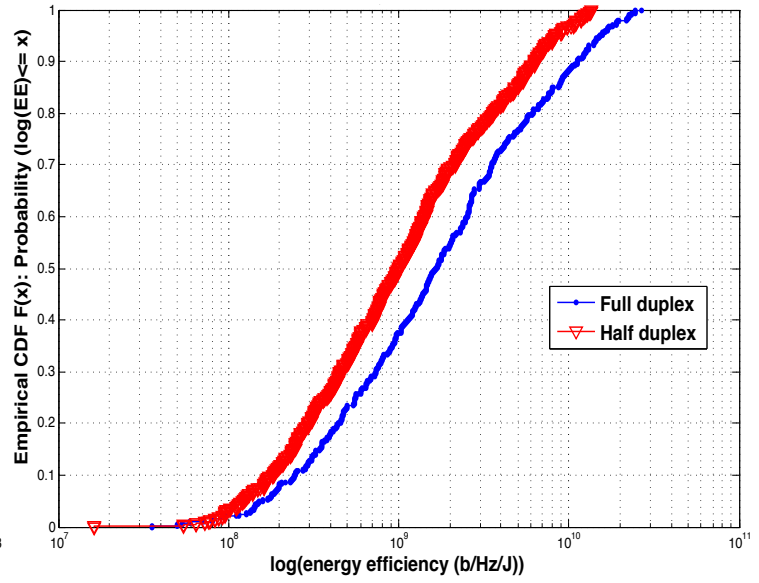

(b)

Figure 3: Energy efficiency of a system with 27 cells, each with radius of $20 \mathrm{~m}$ : a) linear scale, b) normalized dB scale

\section{Simulation Results and Discussions}

In this section, we show the results of a simulation experiment conducted on a cellular network whose architecture is similar to that in Fig(1). We simulated a full-duplex transmission scheme and a half-duplex transmission scheme under same conditions.

If users' target SINRs are feasible, each user in both scenarios will be able to transmit with the required throughput. Therefore, the spectral efficiency in the fullduplex system will be twice of that in the half-duplex system because the FD system requires half the bandwidth. However, this merit comes with a cost; full-duplex system must radiate more power to offset the effect of large amount of interference compared to that in half-duplex system. Therefore, to capture the net efficiency, we propose to use the energy efficiency (EE) in terms of bits/Hz/Joule, which is ratio of the spectral efficiency to the radiated power.

Figures 3 through 5 show the empirical cumulative distribution function (CDF) of the energy efficiency in bits/Hz/Joule for both transmission schemes: half-duplex and full-duplex. In all figures, the full-duplex scheme generally outperforms the halfduplex scheme. Furthermore, in worst case scenarios (the lower tail), where users happen to be in bad locations, both schemes will radiate more power to achieve the required QoS. However, in these bad moments, the full-duplex requires to radiate even more power because as the interference increases in the system, the outcome

of increasing the power diminishes (i.e., the slope of the energy efficiency curve 
International Journal of Computer Networks \& Communications (IJCNC) Vol.10, No.5, September 2018

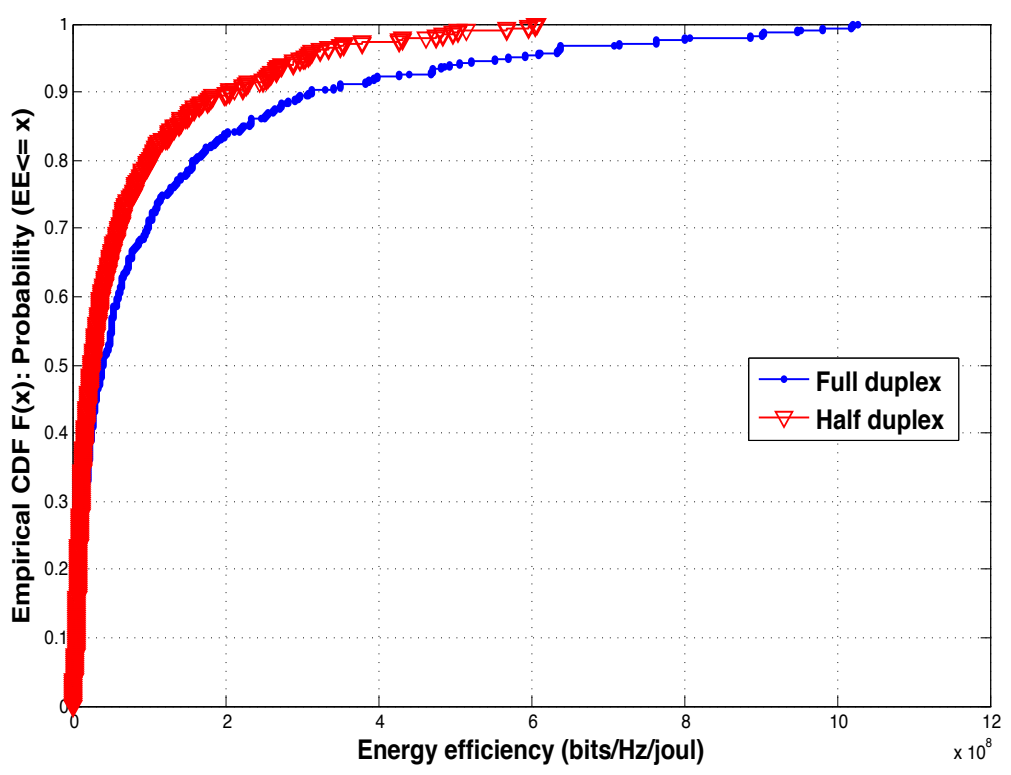

Figure 4: Energy efficiency of a system with 27 cells, each with radius of 50 meter

decreases with respect to the transmit power). On the other hand, as the users move to more favourable locations, the energy efficiency of the full-duplex scheme increasingly outperforms that of half-duplex. This is clearly noticeable from the increasing gap between the CDFs as the EE increases.

In the ideal single-cell system, the ratio of the energy efficiency in full-duplex to that in half-duplex (i.e., $\frac{E E_{F D}}{E E_{H D}}$ ) is theoretically supposed to approach 2 . In the simulated multi-cell system, however, we got an average ratio of about 1.47 . This low ratio (i.e., $\frac{E E_{F D}}{E E_{H D}}=1.47$ ) is due to the large amount of inter-cell interference generated in full-duplex system compared to that in half-duplex system. The average ratio would have been increased had worst located users operated in half-duplex mode; thereby considerably reducing the amount of interference in the network. This result suggests the following:

- as users move to better locations, the ratio $\frac{E E_{F D}}{E E_{H D}}$ increases and approaches 2 .

- operating completely in full-duplex mode for all cells at all times is not always a wise decision, rather, a combination of both modes over the cells (hybrid mode) might be a better choice.

- the transmission mode (half or full) is a new dimension into the problem of radio resource management $(\mathrm{RRM})$. In other words, the RRM algorithm should 
International Journal of Computer Networks \& Communications (IJCNC) Vol.10, No.5, September 2018

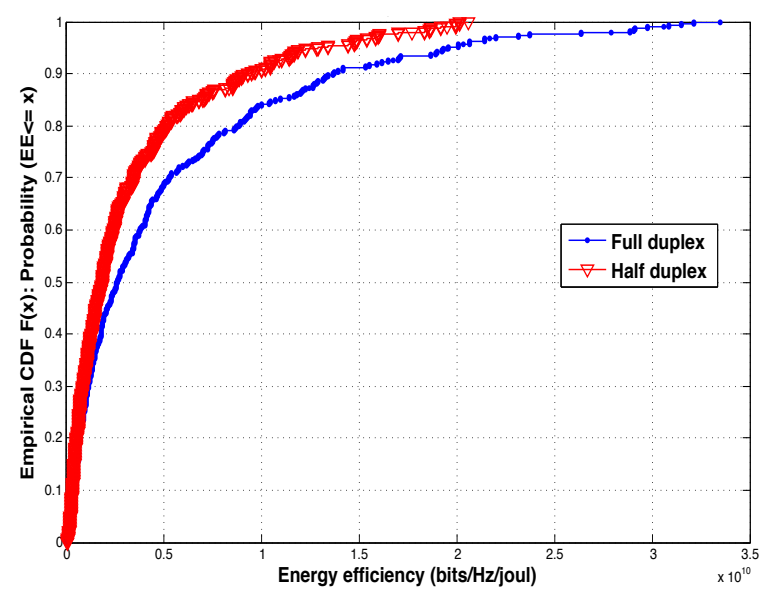

(a)

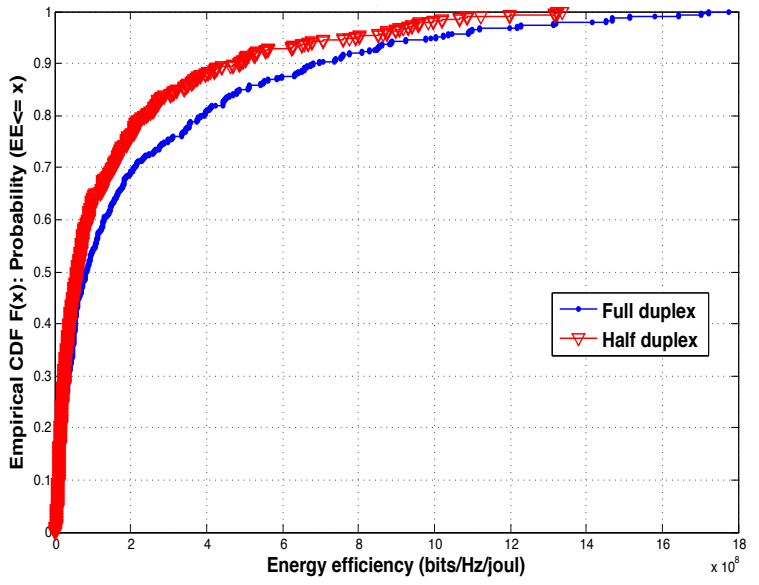

(b)

Figure 5: Energy efficiency of a system with 60 cells: a) 20 meter cell radius, b) 50 meter cell radius

decide the transmission mode in each cell, over each subchannel, and at each time slot.

- in a hybrid mode, as users move to better locations, as the number of cells operating in full-duplex increases.

- certain criteria/threshold on deciding the transmission mode for each cell must be figured out.

\section{Conclusion And Future Work}

The full-duplex communication has attracted enormous attention due to its potential to double the link spectral efficiency. However, co-channel interference is a major challenge that obstructs a successful deployment of full-duplex multi-cell systems. In this paper, we derived the general expression of the SINRs of both uplink and downlink for different scenarios in large cellular SCFD-based networks. Furthermore, we addressed the feasibility of achieving certain QoS requirements in central and distributed setups. It was shown that if the spectral radius of the product of a diagonal matrix and interference channel gains matrix is less than one, then the set of target SINRs is achievable. Moreover, in the case of feasibility, the central achieving policy is the solution of a simple linear equation while the distributed solution can 
International Journal of Computer Networks \& Communications (IJCNC) Vol.10, No.5, September 2018

be found by applying the best response algorithm until reaching to the fixed point. Simulation results show that as users become in better locations, the efficiency of the full-duplex transmission approaches twice the efficiency of half-duplex transmission. On the other hand, when users are in worst locations (i.e., cell edge), the efficiency of full-duplex is indifferent to the half-duplex. Moreover, the hybrid transmission mode a cross the whole network is shown to be the best operational mode. As an extension of this research, we plan to study the case of infeasible target SINRs and find the best policy that maximizes the fairness among all SCFD equipped UEs. In addition, identifying specific criteria for deciding the best transmission mode (full or half ) is still an open problem.

\section{REFERENCES}

[1] A. Goldsmith, Wireless Communication. Cambridge University Press, First edition, 2005.

[2] A. Sabharwal, P. Schniter, D. Guo, D. W. Bliss, S. Rangarajan, and R. Wichman, "In-band full-duplex wireless: Challenges and opportunities," IEEE Journal on Selected Areas in Communications, vol. 32, Sept.. 2014.

[3] E. Everett, A. Sahai, and A. Sabharwal, "Passive self-interference suppression for full-duplex infrastructure nodes," IEEE Trans. Wireless Commun., vol. 13, pp. 680 -694, Feb. 2014.

[4] W. Cheng, X. Zhang, and H. Zhang, "Optimal dynamic power control for fullduplex bidirectional-channel based wireless networks," in Proc. IEEE INFOCOM, pp. 3120-3128, Apr. 2013.

[5] S. Hong, J. Brand, J. Choi, M. Jain, J. Mehlman, S. Katti, and P. Levis, "Applications of self-interference cancellation in $5 \mathrm{~g}$ and beyond," IEEE Communications Magazine, vol. 52, pp. 114-121, Feb. 2014.

[6] J. Jung, H. Roh, J. Kim, H. Kwak, M. Jeong, and J. Park, "Tx leakage cancellation via a microcontroller and high tx-to-rx isolations covering an uhf rfid frequency band of 908 to $914 \mathrm{mhz}$," IEEE Microwave and Wireless Components Letters, vol. 18, no. 10, pp. $710-712,2008$.

[7] J. C. et al., "Achieving single channel, full duplex wireless communication," in Proc. ACM MOBICOM, (Chicago, IL), 2010. 
International Journal of Computer Networks \& Communications (IJCNC) Vol.10, No.5, September 2018

[8] C. Kim, J. Kim, and S. Hong, "A quadrature radar topology with tx leakage canceller for 24-ghz radar applications," IEEE Transactions on Microwave Theory and Techniques, vol. 55, no. 7, pp. 1438-1444, 2007.

[9] D. Bliss, P. Parker, and A. Margetts, "Simultaneous transmission and reception for improved wireless network performance," in Proc. IEEE Statistical Signal Processing Workshop, Aug. 2007.

[10] T. Riibonen, S. Werner, and R. Wichman, "Mitigation of loopback selfinterference in full-duplex mimo relays," IEEE Transactions on Signal Processing, vol. 59, pp. $5983-5993$, Dec. 2011.

[11] T. Snow, C. Fulton, and W. J. Chappell, "Transmit-receive duplexing using digital beamforming system to cancel self interference," IEEE Transactions on Microwave Theory and Techniques, vol. 59, pp. 3494 -3503, Dec. 2011.

[12] M. Duarte, A. Sabharwal, V. Aggarwal, R. Jana, K. Ramakrishnan, C. Rice, and N. Shankaranarayanan, "Design and characterization of a full-duplex multiantenna system for wifi networks," IEEE Transactions on Vehicular Technology, vol. 63, pp. 1160 - 1177, March 2014.

[13] J. Sangiamwong, T. Asai, J. Hagiwara, Y. Okumura, and T. Ohya, "Joint multifilter design for full-duplex mu-mimo relaying," in Proc. IEEE Vehicular Technology Conference, VTC'09., pp. 1-5, Spring 2009.

[14] T. Riihonen, S. Werner, and R. Wichman, "Spatial loop interference suppression in full-duplex mimo relays," in Proc. Asilomar Conference on Signals, Systems and Computers, pp. 1508-1512, Nov. 2009.

[15] D. Bharadia, E. McMilin, and S. Katti, "Full duplex radios," in Proc. ACM SIGCOMM, (Hong Kong), 2013.

[16] P. Larsson and M. Prytz, "Mimo on-frequency repeater with self-interference cancellation and mitigation," in Proc. IEEE 69th Vehicular Technology Conference, VTC'09, pp. 1-5, Spring 2009.

[17] D. Nguyen and M. Krunz, "Be responsible: A novel communications scheme for full-duplex mimo radios," in Proc. IEEE INFOCOM Conference, Kowloon, Hong Kong, April 2015. 
International Journal of Computer Networks \& Communications (IJCNC) Vol.10, No.5, September 2018

[18] A. Cirik, R. Wang, Y. Rong, and Y. Hua, "Mse-based transceiver designs for full-duplex mimo cognitive radios," IEEE Trans. Commun., vol. 63, pp. 20562070, June 2015.

[19] J. Andrews, "Interference cancellation for cellular systems: a contemporary overview," IEEE Wireless Comm., vol. 12, pp. 19-29, Apr. 2005.

[20] A. Amiri, "Optimal bandwidth allocation with bandwidth reservation and adaptation in wireless communication networks," International Journal of Computer Networks Communications (IJCNC), vol. 8, pp. 21-33, Jan. 2016.

[21] B. P. Day, A. R. Margetts, D. W. Bliss, and P. Schniter, "Full-duplex mimo relaying: achievable rates under limited dynamic range," IEEE J. Sel. Areas Commun., vol. 30, pp. 1541-1553, Sep. 2012.

[22] S. Goyal, P. Liu, S. Hua, and S. Panwar, "Analyzing a full-duplex cellular system," in Proc. Annual Conference on Information Sciences and Systems (CISS), pp. 1-6, March 2013.

[23] K. Kinoshita, M. Nakagawa, K. Kawano, and K. Murakami, "An efficient spectrum sharing method based on genetic algorithm in heterogeneous wireless network," International Journal of Computer Networks Communications (IJCNC), vol. 6, pp. 145-156, Sep. 2014.

[24] F.-B. Li, P.-X. Li, Y.-F. Yang, and H. Li, "Game theory based interference control and power control for d2d communication in cellular networks," International Journal of Computer Networks Communications (IJCNC), vol. 8, pp. 27-40, Sep. 2016.

[25] T. Riihonen, S. Werner, R. Wichman, and E. Zacarias, "On the feasibility of full-duplex relaying in the presence of loop interference," in Proc. IEEE 10th Workshop on Signal Processing Advances in Wireless Communications, pp. 275279, June 2009.

[26] B. Yin, M. Wu, C. Studer, J. Cavallaro, and J. Lilleberg, "Full-duplex in largescale wireless systems," in 2013 Asilomar Conference on Signals, Systems and Computers, pp. 1623-1627, Nov. 2013.

[27] R. Horn and C. Johnson, Matrix analysis. Cambridge University Press, 1985.

[28] R. Yates, "A framework for uplink power control in cellular radio systems," IEEE J. Sel. Areas Commun., vol. 13, pp. 1341-1347, 1995. 
International Journal of Computer Networks \& Communications (IJCNC) Vol.10, No.5, September 2018

\section{Author}

Ali Al-Zahrani received his B.S. in Electrical Engineering with honor from King Fahad University of Petrolume and Minirals (KFUPM), Dhahran, Saudi Arabia, in 2002. He further received his M.S. and $\mathrm{PhD}$ in Electrical and Computer Engineering from Carleton University, Ottawa, Canada in 2010 and 2015, respectively. Ali is now an Assistant Professor in the Department of Electrical and Computer Engineering at the University of Jeddah, Saudi Arabia. He worked as an engineer at the Saudi Basic Industries Corporation (SABIC) from 2002 to 2007. His research interests include radio resource allocation, interference management in wireless communication systems and massive MIMO.

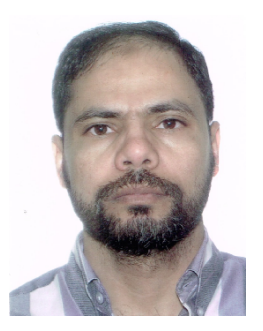

